\title{
Calabi-Yau Manifolds as Complete Intersections in Products of Complex Projective Spaces
}

\author{
Paul Green ${ }^{1}$ and Tristan Hübsch ${ }^{2}$ \\ ${ }^{1}$ Department of Mathematics, University of Maryland, College Park, MD 20742, USA \\ ${ }^{2}$ Department of Physics and Astronomy, University of Maryland, College Park, MD 20742, USA
}

\begin{abstract}
We consider constructions of manifolds with $S U(3)$ holonomy as embedded in products of complex projective spaces by imposing certain homogeneous holomorphic constraints. We prove that every such construction leads to one deformation class of manifolds with $S U(3)$ holonomy. For a subset of these manifolds we prove simple connectedness, address the problem of calculating the second Betti number and explicitly calculate it for a class of constructions. This establishes a very wide class of manifolds with $S U(3)$ holonomy, that can give rise to yet many more constructions via dividing out the action of suitably chosen discrete groups. Some of the examples studied may yield phenomenologically acceptable models.
\end{abstract}

\section{Introduction}

The existence of Ricci-flat Kähler manifolds was conjectured almost thirty years ago [1], but was proven only twenty years after that [2]. Manifolds of this type (generally called Calabi-Yau) of complex dimension $n$ have $S U(n)$ holonomy and one covariantly constant everywhere non-vanishing and non-zero holomorphic $n$-form.

Owing to these features, Calabi-Yau manifolds of complex dimension 3 seem to have an immense impact on the analysis [3] of the phenomenology of superstring theories [4], hopefully leading to a phenomenologically acceptable model of unification of the known basic interactions and matter. Since the invariants of the complex structure of Calabi-Yau manifolds link tightly to the parameters of the physical models [3,5], explicit constructions and computations of the corresponding invariants are necessary in this approach in order to make contact between the superstring theories and the real world of experiments.

In the usual analysis of the phenomenology [3], one requires a manifold of Euler character \pm 6 , multiply connected, with a preferrably big discrete structure group. The requirements on the set of discrete symmetries of the manifold as well as its structure group are rather model dependent though. The second Betti 
number $b_{2}$ is also very important since the $(1,1)$-Hodge number turns out to be the number of Higgs fields, and on Calabi-Yau manifolds there are no harmonic $(2,0)$-, or $(0,2)$-forms and so $b_{2}=b_{1,1}$.

Another approach is also found in the literature [6] where one only required the Euler character to be \pm 4 and the manifold to be multiply connected. Other details of the topology and geometry do not seem to be relevant and a few explicitly constructed examples already exist [7].

It has been also proposed that some Calabi-Yau manifolds may admit stable holomorphic vector bundles with the $S U(4)$ or $S U(5)$ structure groups [8]. Whether such manifolds can be constructed is still not clear, but a better understanding of the constructions of Calabi-Yau manifolds and related computations should certainly help.

A few Calabi-Yau manifolds were constructed as algebraic varieties in $C P^{n}$, products $C P^{n} \times C P^{m}$, as branched coverings and some "exotic" cases $[3,7,9]$. A wide class of constructions was presented [10] in a more systematic attempt, exhausting the possibilities of constructing Calabi-Yau manifolds as algebraic varieties in $m$-fold products of $C P^{n}$ 's. Still, no classification of Calabi-Yau manifolds exists and moreover, the finiteness of their number is also an open question. In most of these constructions, simply-connected algebraic varieties embedded in (products of) $C P^{n}$ 's, and further Calabi-Yau manifolds were obtained by dividing the free action of a discrete group (or blowing up the singular points if any).

Following this spirit, we shall focus here only on Calabi-Yau manifolds embedded in products of $C P^{n}$ 's. Let $z_{\mu r}$ denote the $\mu^{\text {th }}$ homogeneous coordinate of the $r^{\text {th }} C P_{r}^{n_{r}}$ in $\mathscr{M} \equiv C P_{1}^{n_{1}} \times \ldots \times C P_{m}^{n_{m}}$. Then we shall impose the vanishing of a set of homogeneous holomorphic polynomials (hereafter constraints):

$$
I^{a} \equiv \sum_{r=1}^{m} \alpha_{a}^{\mu r}\left(z_{\mu r}\right)^{q_{a}^{r}}=0
$$

where summation over $\mu$ is understood for the homogeneous coordinates if each $C P$ separately and the index $a$ enumerates the constraints, taking values from 1 through $\eta=\left(\sum_{r=1}^{m} n_{r}\right)-3$, to yield a complex three-dimensional submanifold $\mathscr{N}$ of $\mathscr{M} . \alpha_{a}^{\mu r}$ are complex coefficients and $q_{a}^{r}$ is the degree of homogeneity of the $a^{\text {th }}$ constraint in the homogeneous coordinates of $C P_{r}^{n_{r}}$. These constraints must also provide a nowhere vanishing volume-form for the normal bundle:

$$
d I^{1} \wedge \ldots \wedge d I^{\eta} \neq 0
$$

in $\mathscr{N}$. To ensure that the $\mathscr{N}$ is indeed a Calabi-Yau manifold, one has to restrict the matrix $\mathbf{q}$ so as to yield a vanishing first Chern class $[1,2]$. Note that the total Chern class can be evaluated as:

$$
\begin{gathered}
c[\mathcal{N}]=\xi^{-1} \cdot \prod_{r=1}^{m}\left(1+x_{r}\right)^{n_{r}+1}, \\
\xi=\prod_{a=1}^{\eta}\left(1+\sum_{r=1}^{m} q_{a}^{r} x_{r}\right) .
\end{gathered}
$$


The requirement of vanishing first Chern class now reduces to:

$$
c_{1}^{r}=(n+1)-\sum_{a=1}^{\eta} q_{a}^{r}=0, \quad \forall r .
$$

Also, the Euler character is now found as the integral of the third Chern class over $\mathscr{N}$ :

$$
\chi_{E}=\int_{\mathscr{N}} c_{3}[\mathscr{N}]=\int_{\mathscr{M}} \xi \cdot c_{3}[\mathscr{N}]=\left[\xi \cdot c_{3}[\mathscr{N}]\right]_{\mathrm{top}},
$$

where $x_{r}$ denotes the Kähler form of $C P_{r}^{n_{r}}$, its products are wedge products and $[X]_{\text {top }}$ denotes the coefficient of the top $\left[\right.$ i.e. $\left.\left(x_{1}\right)^{n_{1}} \ldots\left(x_{m}\right)^{n_{m}}\right]$ term in the expansion of $X$.

Equation (1) yields a class of algebraic varieties embedded in $\mathscr{M}$, which we call a configuration and note that it may be represented by its matrix $\mathbf{q}$. By definition, we exclude matrices which can be brought into a block-diagonal form such that the off-diagonal blocks vanish, since these would yield varieties having the form of a Cartesian product and not Calabi-Yau manifolds.

The purpose of this paper is threefold and consequently, the organization is as follows: First, in Sect. 2, we give a conclusive proof that all configurations indeed contain non-singular Calabi-Yau manifolds. Next, in Sect. 3 we prove that finitely many configurations contain all the distinct Calabi-Yau manifolds that can be constructed in this way. In Sect. 4 we prove simple connectedness for a subset of configurations and address the problem of the computation of $b_{2}$ of the manifolds considered so far. Even though calculable in principle, it seems to be a difficult problem in general but quite straightforward for some cases, where we list them. Our conclusions are summarized in Sect. 5.

\section{Every Configuration Contains Non-Singular Varieties}

It is fairly obvious that the method of constructing Calabi-Yau manifolds outlined in the Introduction, is capable of yielding a very wide classs of manifolds. Further examples can be found by dividing out the action of a discrete group of symmetries of the constraints and blowing up the singular points if any. Here we shall prove that every possible configuration indeed contains Calabi-Yau manifolds. To do so, we first state and prove two propositions and then apply them to the configurations.

Let $\mathscr{M}$ be a complex manifold and $\mathscr{L}$ a line bundle over $\mathscr{M}$. Let $\chi$ be a holomorphic section of $\mathscr{L}$ and $m$ a point of $\mathscr{M}$. Let $\mathscr{T}_{m}$ be the tangent space to $\mathscr{M}$ at $m$. If $\chi(m)=0$, then $D \chi \in \mathscr{T}_{m}^{*}$ is well defined independent of any connection.

The line bundle $\mathscr{L}$ is called ample if for every $m \in \mathscr{M}$, there is a global holomorphic section of $\mathscr{L}$ which does not vanish at $m$ and for every $m \in \mathscr{M}$ and $\mu \in \mathscr{T}_{m}^{*}$, there is a global holomorphic section $\chi$ with $\chi(m)=0$ and $D \chi(m)=\mu$.

If $\chi(m)=0=D \chi(m), \chi$ is said to have a vanishing one-jet at $m$. It is standard that if $\chi$ does not have a vanishing one-jet at any point of $\mathscr{M}$, then $\chi^{-1}(0)$ is a nonsingular complex submanifold of $\mathscr{M}$.

Proposition 1. If $\mathscr{L}$ is an ample line bundle over $\mathscr{M}$, then a dense open subset of the space of global holomorphic sections of $\mathscr{M}$ has nowhere-vanishing one-jets. 
Proof. Let $\mathscr{M}$ have complex dimension $m$. Let the space of holomorphic sections of $\mathscr{L}$ have complex dimension $k$. For each $p \in \mathscr{M}$, the space of sections with vanishing one-jet at $p$ has dimension $k-m-1$. Let $C P_{p}^{k-m-2}$ be the corresponding projective space. Then there is a holomorphic bundle $\mathscr{K}$ over $\mathscr{M}$ whose fiber at $p$ is $C P_{p}^{k-m-2}$ and whose total dimension as a compact complex manifold is $k-2$. Clearly there is a mapping from $\mathscr{K}$ to $\mathscr{P}^{k-1}$, the projective space of the space of sections of $\mathscr{L}$, whose image consists of all points of $\mathscr{P}^{k-1}$ corresponding to sections whose one-jet vanishes at that one point of $\mathscr{M}$. Since the image of $\mathscr{K}$ is compact and has codimension at least 1 , the proposition follows.

Consider now $f: \mathscr{M} \rightarrow \mathscr{N}$ be a holomorphic map. Let $\mathscr{L}$ be a holomorphic line bundle over $\mathscr{N}$. Then there is an induced holomorphic line bundle, $\mathscr{L}^{f}$, over $\mathscr{M}$ whose fibre over $m \in \mathscr{M}$ may be identified with the fibre of $\mathscr{L}$ over $f(m)$. Moreover, if $\chi$ is a holomorphic section of $\mathscr{L}$, then there is a section $\chi^{f}$ of $\mathscr{L}^{f}$, linear in $\chi$ where $\chi^{f}(m)$ is identified with $\chi(f(m))$.

Proposition 2. For $\mathscr{M}$ and $\mathscr{N}$ compact complex manifolds, if $\mathscr{L}$ is an ample line bundle over $\mathscr{N}$ and $f: \mathscr{M} \rightarrow \mathscr{N}$ is holomorphic, then $\chi^{f}$ has nowhere-vanishing one-jet over $\mathscr{M}$ for $\chi$ in a dense open connected subset of the projective space of holomorphic sections of $\mathscr{L}$.

Proof. It is evident that it $f$ is an immersion $(\operatorname{Rank} d f \equiv \operatorname{dim} \mathscr{M})$ then $\mathscr{L}^{f}$ is again ample, and that if $f$ is a submersion $(\operatorname{Rank} d f \equiv \operatorname{dim} \mathscr{N})$ then $\chi^{f}$ has nowhere vanishing one-jet whenever $\chi$ does.

To prove the general result, we note first that the projective set of sections of $\mathscr{L}^{f}$ with one-jet vanishing somewhere on $\mathscr{M}$ is compact, and therefore closed as a subspace of the projective space of sections of $\mathscr{L}^{f}$. (This is less obvious than in the case when $\mathscr{L}^{f}$ is ample, but may be verified by a routine sequential compactness argument.) Hence the projective space of sections $\chi$ of $\mathscr{L}$ such that $\chi^{f}$ has a vanishing one jet is also compact.

Next we remark that it is standard from stratification theory that $\mathscr{M}$ is the union of countably many submanifolds $\mathscr{M}_{r}$ such that for each $r,\left.f\right|_{\mathscr{M}_{r}}$ is a submersion followed by an immersion. Thus the projective set of sections of $\mathscr{L}$ such that $\chi$ has a vanishing one-jet is compact and also the union of countably many sets of positive complex codimension in the space of all sections of $\mathscr{L}$. Hence the complement of this set is open, dense and connected.

Theorem 1. Every configuration contains non-singular varieties.

Proof. We note that the homogeneous polynomials may be identified with holomorphic sections of suitably chosen line bundles (see below). Thus we start with

$$
\mathscr{M}_{1} \equiv C P_{1}^{n_{1}} \times \ldots \times C P_{m}^{n_{m}}
$$

and impose the constraints iteratively.

$\mathscr{F}$ or the $a^{\text {th }}$ constraint, we choose $\chi_{a}$ to be a section of the holomorphic line bundle $\mathscr{L}_{a} \equiv \prod_{r}\left(h_{r}\right)^{q_{a}}$, where $h_{r}$ is the hyperplane bundle over $C P_{r}^{n_{r}}$ and the product is the tensor product. The manifold we want to construct is $\bigcap_{a} \chi_{a}^{-1}(0)$. We prove iteratively that the $\chi_{a}$ can be chosen so that $\bigcap_{a} \chi_{a}^{-1}(0)$ is non-singular. 
Let us define:

$$
\mathscr{N}_{a}=\prod_{q_{a}^{r}>0} C P_{r}^{n_{r}}, \quad \mathscr{M}_{a}=\bigcap_{b<a} \chi_{b}^{-1}(0)
$$

We note that $\mathscr{L}_{a}$ is ample over $\mathscr{N}_{a}$, and apply Proposition 2 to $\mathscr{L}_{a}$ and $f_{a}: \mathscr{M}_{a} \rightarrow \mathscr{N}_{a}$ where $f_{a}$ is the inclusion of $M_{a}$ in $\prod_{r} C P_{r}^{n_{r}}$ followed by the projection on $\mathscr{N}_{a}$. The induction starts with $\mathscr{M}_{1}=\prod C P_{r}^{n_{r}}$ and proceeds via the observation that $\mathscr{M}_{a+1}$ may be identified with $\left(\chi^{f_{a}}\right)^{-1}(0)$.

Remark. Since the set of choices of constraints leading to non-singular CalabiYau manifolds is an open connected subset of the projective space of all possible choices of constraints for a given configuration, it follows that all Calabi-Yau manifolds belonging to a given configuration are equivalent by deformation.

\section{Sufficiency of Finitely Many Configurations}

Let us first concentrate on the infinite sequence of configurations where the CalabiYau manifold is embedded in an $m$-fold product of $C P^{1}$ 's [10]. The matrix $q$ must satisfy Eq. (5) that ensures the vanishing of the first Chern class, so:

$$
\sum_{a=1}^{\eta} q_{a}^{r}=n+1, \quad \forall r
$$

Next we note that a bilinear constraint reduces $C P^{1} \times C P^{1}$ to the "diagonal" $C P^{1}$. On the other hand, a quadratic constraint involving only one $C P^{1}$ would "saturate" Eq. (7) and lead to a product structure of the submanifold. Thus in order to obtain a configuration that cannot be reduced to a previous one, we conclude:

$$
\sum_{r=1}^{m} q_{a}^{r} \geqq 3, \quad \forall a .
$$

Performing now the "other" summations on the last two relations and comparing results, one obtains that for $m>9$, the configuration can always be reduced to one with $m \leqq 9$. Thus the infinite sequence of configurations indeed yields only a finite number of non-reducible configurations, and thus by the proof in Sect. 2, to a finite number of deformation classes of Calabi-Yau manifolds.

This argument can actually be generalized so as to include all configurations. To do so, we first define a configuration to be minimal if it contains Calabi-Yau manifolds which do not belong to any configuration of lower total dimension.

To show that there are finitely many minimal configurations, let us consider:

$$
C P^{1} \times \ldots \times C P^{1} \times C P^{n_{1}} \times \ldots \times C P^{n_{p}}
$$

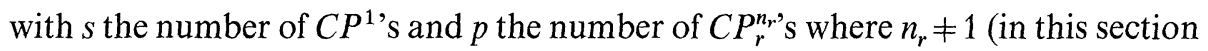
only). For future convenience, we also define:

$$
\alpha=\sum_{r=1}^{p}\left(n_{r}-1\right), \Rightarrow p \leqq \alpha,
$$

where the inequality follows since $n_{r} \geqq 2, \forall r$. 
The number of constraints necessary to obtain a variety of complex dimension three is then:

$$
s+\sum_{r=1}^{p} n_{r}-3=s+p+\alpha-3
$$

and the total degree of all constraints is:

$$
2 s+\sum_{r=1}^{p}\left(n_{r}+1\right)=2 s+2 p+\alpha
$$

in order to satisfy Eq. (7). Since the constraints are at least of degree two (linear constraints are trivial since they replace $C P^{n}$ by $C P^{n-1}$ ), it follows that:

$$
2 s+2 p+\alpha \geqq 2(s+p+\alpha-3), \Rightarrow \alpha \leqq 6,
$$

and hence an upper limit on the number of $C P_{r}^{n_{r}}$ 's:

$$
p \leqq \alpha \leqq 6 .
$$

By the same argument that led to Eq. (8), we note that if a constraint is bilinear and involves a $C P^{1}$, it must involve a $C P_{r}^{n_{r}}$ as well. Let the number of such constraints be $a$ (which is also the total degree of these constraints in coordinates of the $C P^{1}$ 's and also for the $C P_{r}^{n_{r}}$ 's). For constraints of degree $>2$, let $b$ denote the total degree (summed over the constraints) in the coordinates of $C P^{1}$ 's only. Then, obviously, $a+b=2 s$ to ensure Eq. (7) for the $C P^{1}$ 's. However, since the bilinear constraints must involve a $C P_{r}^{n_{r}}$ as well:

Furthermore, note that: $\quad a \leqq 2 p+\alpha$.

$$
\sum_{\text {all constr. }}(\text { degrees }-2)=(2 s+2 p+\alpha)-2(s+p+\alpha-3)=6-\alpha,
$$

is the number of constraints with degree $>2$, if they are of degree precisely three. On the other hand, for biggest $b$, these constraints should involve only $C P^{1}$ 's and so:

$$
b \leqq 3(6-\alpha) \text {. }
$$

Since $a+b=2 s$ it follows that the number of $C P^{1}$ 's is limited as well:

$$
s \leqq 9-(\alpha-p) \leqq 9,
$$

where the last inequality follows from relation 13 .

Since both the number of $C P^{1}$ 's and the number of $C P_{r}^{n_{r}}$ 's is bounded from above by relations 13 and 16 respectively, the number of possible minimal configurations is finite. Note that the number of possibilities for minimal configurations are further reduced by the observation that a quadratic complex curve in $C P^{2}$ is a $C P^{1}$, and a quadratic surface in $C P^{3}$ is $C P^{1} \times C P^{1}$.

\section{Computation of the Betti Numbers}

As mentioned in the Introduction, the knowledge of $b_{2}$ is very important for the physical application of the Calabi-Yau manifolds and now we address this problem. 
We rely on the following generalization of the Lefschetz Hyperplane Theorem (LHT):

Let $\mathscr{L}$ be a positive holomorphic line bundle over the n-dimensional complex manifold $\mathscr{M}$, and $\chi$ be a holomorphic section of $\mathscr{L}$. Then:

$$
\pi_{i}\left(\mathscr{M}, \chi^{-1}(0)\right)=0, \text { for } i \leqq n .
$$

The proof of LHT for the case when $\chi^{-1}(0)$ is non-singular can be found in [11] but it is easily modified to accommodate the singular case as well.

As a consequence of LHT the inclusion of $\chi^{-1}(0)$ in $\mathscr{M}$ induces isomorphisms in homotopy in dimensions $<n-1$ and an epimorphism in dimension $n$. We note that, in the notation of Sect. $2, \mathscr{L}_{k}$ is positive over $\mathscr{N}_{k}$. However, $\mathscr{L}_{k}^{f_{k}}$ is in general not positive over $\mathscr{M}_{k}$. Note also that $b_{2}$ equals the $(1,1)$-Hodge number, since a two form decomposes into a $(2,0)$, a $(1,1)$ and a $(0,2)$-form under Hodge decomposition, but there are no harmonic $(2,0)$ or $(0,2)$-forms on a Calabi-Yau manifold. We shall demonstrate our method on a number of examples and also indicate its limitations.

Let us define:

$$
D_{k}:=\left\{r \mid q_{a}^{r}>0\right\} .
$$

We will call a configuration favourable if the constraints can be ordered so that:

$$
\text { for } k^{\prime}<k \text { : either } D_{k^{\prime}} \cap D_{k}=\emptyset \text {, or } D_{k^{\prime}} \subset D_{k} \text {. }
$$

Theorem 2. (i) A Calabi-Yau manifold belonging to a favourable configuration is simply connected.

(ii) A Calabi-Yau manifold belonging to a minimal favourable configuration has the second Betti number at least equal to the number of CP's.

Proof. We proceed by induction using the notation of the proof of Theorem 1. Clearly, $\mathscr{M}_{1}$ satisfies both (i) and (ii). By the hypothesis that the configuration is favourable, we may write

$$
\mathscr{M}_{a}=\mathscr{Q}_{a} \times \mathscr{R}_{a}, \quad \mathscr{R}_{a} \subset \mathscr{N}_{a},
$$

and note that $f_{a}$ is the projection on $\mathscr{R}_{a}$ followed by the inclusion. Then

$$
\mathscr{M}_{a+1}=\mathscr{Q}_{a} \times\left(\mathscr{R}_{a} \cap \varphi_{a}^{-1}(0)\right),
$$

where $\varphi_{a}^{-1}(0)$ is a section of $\mathscr{L}_{a}$. We consider now three cases:

$\operatorname{dim} \mathscr{R}_{a}=1$. This case cannot occur because the total degree of all the constraints involving $\mathscr{N}_{a}$ alone is at most $2 \operatorname{dim} \mathscr{N}_{a}$. Since each constraint is at least quadratic, the $a^{\text {th }}$ constraint would exhaust the constraints on $\mathscr{N}_{a}$, bringing $\mathbf{q}$ to a blockdiagonal form that contradicts the definition of a configuration.

$\operatorname{dim} \mathscr{R}_{a}=2$. Now $\mathscr{R}_{a} \cap \varphi_{a}^{-1}(0)$ is a curve. The only possibilities consistent with Eq. (5) in the definition of a configuration are a Torus or a $C P^{1}$. If $\mathscr{R}_{a} \cap \varphi_{a}^{-1}(0)$ were a Torus, it would be possible to bring $\mathbf{q}$ to a block-diagonal form that is excluded by its definition. In the case of a $C P^{1}, \mathscr{M}_{a+1}$ would be simply connected, but the original configuration would not be minimal since it could be reduced by replacing $\mathscr{N}_{a}$ by $\mathscr{R}_{a} \cap \varphi_{a}^{-1}(0)$. 
$\operatorname{dim} \mathscr{R}_{a} \geqq 3$. We invoke the LHT to conclude that $\mathscr{R}_{a} \cap \varphi_{a}^{-1}(0)$ is simply connected and has $b_{2}$ greater than or equal to $b_{2}\left(\mathscr{R}_{a}\right)$.

This completes the induction.

Remark. The statement (ii) cannot be made stronger since, as we shortly demonstrate, there are examples with $b_{2}$ greater than the number of $C P$ 's.

Now we turn to the configurations presented in [10] adopting the notation where the first column in the matrix denotes the dimensions of the CP's and the rest of the matrix are the degrees of homogeneities of the constraints with respect to each of the $C P$ 's. We label the Euler character by the subscript and $b_{2}$ by the superscript of the configuration matrix. We start with those embedded in $C P^{n} \times C P^{n}$. Note that in:

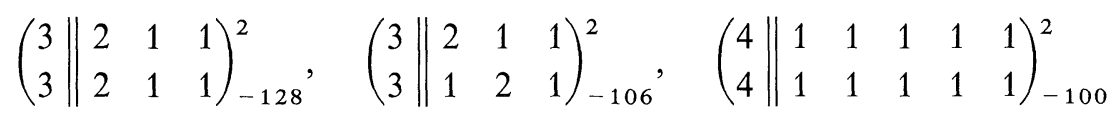

all the constraints involve both $C P^{n}$ 's so LHT applies immediately and the sequence of Betti numbers (which we denote by b) agrees with that of $C P^{n} \times C P^{n}$ up through $b_{2}$ (since the varieties are of complex dimension three). Since for every $C P^{n}$ the odd Betti numbers vanish and the even ones are 1, and:

$$
b_{i}[\mathscr{M} \times \mathscr{N}]=\sum_{j+k=i} b_{j}[\mathscr{M}] b_{k}[\mathscr{N}]
$$

we obtain that $b_{2}=2$ for the above configurations, as indicated by the exponents.

Next we observe that the first constraint in:

$$
\left(\begin{array}{l||lllll}
4 & 2 & 0 & 1 & 1 & 1 \\
4 & 0 & 2 & 1 & 1 & 1
\end{array}\right)_{-88}^{2}
$$

defines an algebraic variety of complex dimension three, embedded in $C P^{4}$, and similarly the second one. Thus $\mathbf{b}$ of these varieties agrees with $C P^{4}$ up to $b_{2}$, and since the other three constraints involve both the $C P^{4}$ 's, LHT applies directly yielding $b_{2}$ for the Calabi-Yau manifold in the above configuration.

It is straightforward to verify that e.g. a quadric in $C P^{3}$ leads to a surface with $b_{2}=2$ (and $\chi_{E}=4$ ) yielding $b_{2}=3$ for the configuration:

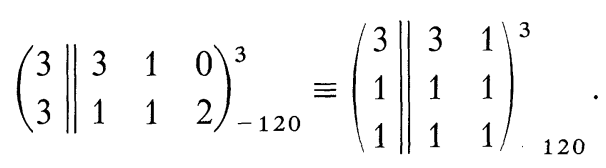

The identity follows from $(3 \| 2) \equiv C P^{1} \times C P^{1}$ as mentioned in Sect. 3 .

Note that owing to identities mentioned above we have:

$$
\left(\begin{array}{l||lll}
3 \\
3
\end{array} \| \begin{array}{lll}
2 & 0 & 2 \\
0 & 2 & 2
\end{array}\right)_{-128}^{4} \equiv\left(\begin{array}{l}
2 \\
2 \\
2 \\
2
\end{array} \| \begin{array}{lllll}
2 & 1 & 0 & 0 & 0 \\
0 & 1 & 2 & 0 & 0 \\
0 & 1 & 0 & 2 & 0 \\
0 & 1 & 0 & 0 & 2
\end{array}\right)_{-128}^{4} \equiv\left(\begin{array}{l}
1 \\
1 \\
1 \\
1
\end{array}\left\|\left.\right|_{-128} ^{2}\right\|_{2}^{4}\right.
$$


Using this technique, we obtain $b_{2}$ for twelve more configurations and indicate it by the exponent:

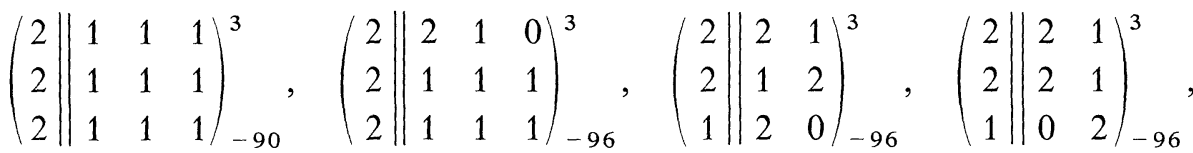

$$
\begin{aligned}
& \left(\begin{array}{l||l}
3 \\
1 \\
1
\end{array} \mid \begin{array}{ll}
2 & 2 \\
1 & 1 \\
1 & 1
\end{array}\right)_{-104}^{3}, \quad\left(\begin{array}{l}
2 \\
2 \\
1
\end{array} \| \begin{array}{ll}
2 & 1 \\
2 & 1 \\
2 & 0
\end{array}\right)_{-132}^{3},\left(\begin{array}{l}
2 \\
1 \\
1
\end{array} \| \begin{array}{l}
3 \\
2 \\
2
\end{array}\right)_{-144}^{3}, \\
& \left(\begin{array}{l||lll}
2 \\
2 \\
2
\end{array} \mid \begin{array}{lll}
3 & 0 & 0 \\
1 & 1 & 1 \\
1 & 1 & 1
\end{array}\right)_{-108}^{5}, \quad\left(\begin{array}{l||l}
3 \\
1 \\
1
\end{array} \| \begin{array}{ll}
3 & 1 \\
0 & 2 \\
0 & 2
\end{array}\right)_{-48}^{9}, \quad\left(\begin{array}{l||lllll}
4 & 2 & 0 & 0 & 1 \\
4 & \| & 0 & 2 & 2 & 1
\end{array}\right)_{-32}^{12}, \\
& \left(\begin{array}{l}
1 \\
1 \\
2 \\
2
\end{array} \|\left(\begin{array}{lll}
2 & 0 & 0 \\
2 & 0 & 0 \\
1 & 1 & 1 \\
1 & 1 & 1
\end{array}\right)_{-96}^{6}, \quad\left(\begin{array}{l}
2 \\
2 \\
2 \\
2
\end{array} \| \begin{array}{lllll}
1 & 1 & 1 & 0 & 0 \\
1 & 1 & 1 & 0 & 0 \\
1 & 0 & 0 & 1 & 1 \\
1 & 0 & 0 & 1 & 1
\end{array}\right)_{-72}^{8} .\right.
\end{aligned}
$$

Note that $b_{2}$ is much larger then the number of $C P$ 's for the last five cases, illustrating the remark following Theorem 2.

\section{Conclusions}

We have proven that every configuration indeed contains one deformation class of Calabi-Yau manifolds. This clearly gives rise to an immense number of possible constructions and being a systematic approach may eventually shed some light on the classification of these manifolds. We have also proven the simple connectedness of a subset of manifolds obtained this way and computed $b_{2}$ for some of them.

There is, however, a still larger class of non-favourable configurations to which our Theorem 2 does not apply. Some progress can be made in computing the Betti numbers of the intersections of hypersurfaces where constraints "overlap" by applying the LHT to the singular hypersurface corresponding to the tensor product of two constraints whose null set is the union of the hypersurfaces, and computing with Mayer-Vietoris sequences.

Let us finally remark that a number of configurations contain manifolds the Euler character of which is properly divisible by 6 (favoured in the usual approach to construct phenomenologically acceptable models) and thus may lead to constructions applicable in supergrandunified model building. The second Betti number of these examples also seems to fall in the phenomenologically favourable category. 


\section{References}

1. Calabi, E.: In: Algebraic geometry and topology: A symposium in honour of S. Lefschetz. Princeton, NJ: Princeton University Press 1957, p. 78

2. Yau, S.-T.: Proc. Natl. Acad. Sci. 74, 1798 (1977)

3. Candelas, P., Horowitz, G.T., Strominger, A., Witten, E.: Vacuum configurations for superstrings. Nucl. Phys. B 258, 46 (1985)

4. Schwarz, J.H.: Superstrings. Singapore: World Scientific 1985

5. Witten, E.: Symmetry breaking patterns in superstring models. Nucl. Phys. B 258, 75 (1985)

6. Hübsch, T., Nishino, H., Pati, J.C.: Do superstrings lead to quarks or preons? Phys. Lett. $163 \mathrm{~B}, 111$ (1985)

7. Strominger, A., Witten, E.: New manifolds for superstring compactification. Commun. Math. Phys. 101, 341 (1985)

8. Donaldson, S.: An application of gauge theory to four dimensional topology. J. Differ. Geom. 18, 269 (1983)

Freed, D.S., Uhlenbeck, K.K.: Instantons and four-manifolds. Berlin, Heidelberg, New York: Springer 1984

Strominger, A.: Superstrings with torsion. University of California Report NSF-ITP-86-16 Witten, E.: New issues in manifolds with SU(3) holonomy. Nucl. Phys. B 268, 79 (1986)

9. Yau, S.-T.: In: Proc. of symposium on anomalies, geometry, topology, Bardeen, W.A., White, A.R. (eds.), Singapore: World Scientific 1985

10. Hübsch, T.: Calabi-Yau manifolds - motivations and constructions. Commun. Math. Phys. (to appear)

11. Bott, R.: On a theorem of Lefschetz. Mich. Math. J. 6, 211 (1959)

Communicated by L. Alvarez-Gaumé

Received June 6, 1986 